\title{
A Generalized Streamline Method to Predict Reservoir Flow
}

\author{
Blunt M. J., Liu K., Thiele M. R.
}

Stanford University,

Dept. of Petroleum Engineering, U. S. A.

\begin{abstract}
Copyright 1995, Steering Committee of the European IOR - Symposium.
This paper was presented at the 8th. European IOR - Symposium in Vienna. Austria, May 16 - 17, 1995

This paper was solected for presentation by the Steering Committee, following review of information contained in an abstract submitted by the author(s). The paper, as presented has not been reviewed by the Steering Committee.
\end{abstract}

\begin{abstract}
We present a generalized streamline method that is able to model flow in porous media-including gravity, compositional effects and dispersion in three dimensions. First we describe the theory and discuss the approximations of the method. Then we compare the predictions using the streamline technique against high resolution numerical simulation. In cases where the principal flow directions are governed by the pattern of permeability, the agreement with conventional simulation is excellent, and the streamline method produces predictions free from numerical diffusion in two to four orders of magnitude less computer time. Last we present a large three-dimensional example and discuss the use of the method for reliable performance prediction for reservoirs where an ensemble of fine scale geostatistical descriptions of the permeability and porosity is known.
\end{abstract}

\section{Introduction}

Current geostatistical techniques generate detailed descriptions of reservoir permeability and porosity that may contain tens or hundreds of millions of grid blocks. To predict the uncertainty in reservoir performance due to the uncertainties in the geology would require flow simulations of the same size on many geostatistical realizations. However, conventional- simulation is limited to around half a million grid cells for miscible displacements [Christie et al., 1993; Tchelepi and Orr, 1994]. Simulation of more complex displacements, such as gas injection with compositional effects, are typically limited to small studies containing only a few thousand grid blocks [Blunt et al., 1994]. Because of numerical diffusion, modeling the correct sequence of fronts in a compositional displacement requires of order 100 grid blocks in one direction. Combined with reservoir heterogeneity on many length scales and multiple wells, this means that simulations of many million grid blocks are required in three dimensions - well beyond the capacity of present models. To overcome these difficulties, upscaling methods have been developed that aim to reproduce the fine scale behavior by appropriately averaged functions on a coarse scale. However, as yet there is no proven method that can start with an ensemble of detailed reservoir descriptions and predict reservoir performance and its uncertainty, while adequately representing the major reservoir flow units, phase behavior and the details of the fluid advance. In this paper we present a direct method that offers the promise to estimate recovery at the field scale on models containing millions of grid blocks.

A streamline is a path that follows the instantaneous velocity field. A streamtube is bounded by streamlines and carries a fixed volume of fluid flux. In this paper we will discuss a method where streamlines are followed through a heterogeneous domain. The idea is equivalent to streamtube methods, and streamline and streamtube will be used interchangeably here to describe the various methods. For tracer flows the velocity field does not change with time, and hence a streamline follows the particle pathway if there is no molecular diffusion or sub-gridblock-scale dispersion. The use of streamlines to describe single phase flow is 
standard in the theory of flow in porous media [Bear, 1972]. Streamline methods to find approximate solutions for multiphase flows started with the work of Higgins and Leighton [1962a; 1962b]. To accommodate changes in flow velocity, Higgins and Leighton changed the flux along each streamtube as the displacement proceeded. This gave excellent predictions of unstable waterfloods in a quarter five spot. Their work has been extended by many authors including [Emanuel et al., 1989; Hewett and Behrens, 1991; King et al., 1993; Lake et al., 1981; Renard, 1990]. The streamtube method is a good approximation when the flow direction is dominated by the permeability and the velocity field does not change greatly with time.

One of the most successful applications of streamtubes used a hybrid method to predict reservoir performance [Emanuel et al., 1989; Hewett and Behrens, 1991; Lake et al., 1981]. Effective fractional flows were computed from conventional fine grid simulation of a representative vertical cross-section of the reservoir. Then streamtubes were calculated areally accounting for the detail of the reservoir boundary and well configurations. A one-dimensional solution, consistent with the computed fractional flow, was then propagated along each tube using the Higgins and Leighton method. Excellent matches for nine field cases, including a $\mathrm{CO}_{2}$ flood, were reported using this method [Emanuel et al., 1989].

More recently, a streamtube method has been proposed where the tubes are periodically recalculated during the displacement [Thiele et al., 1994a; Thiele et al., 1994b; Thiele et al., 1995]. This has been shown to account for the varying flow field more accurately than the Higgins and Leighton technique, and has been used to predict two-dimensional tracer, first-contact miscible, immiscible, and compositional displacements. In all cases the results were in good agreement with conventional numerical simulation and computed the flow up to 10,000 times faster.

The reason why the method of Thiele et. al. works is that in heterogeneous reservoirs, the principal flow paths are controlled by the permeability and along each flow path the displacement can be described by a representative one-dimensional solution. The main advantage of the technique is that the streamtubes only need to be recalculated about ten times to compute the flow well beyond breakthrough, resulting in a speed-up of 3-4 orders of magnitude compared to conventional simulation.

In this paper we extend this work by developing a generalized streamline method. We show that this method is free of the restrictions of previous streamtube methods: the tracing of streamlines is no more difficult in three dimensions than two, whereas the computation of three- dimensional streamtubes, although possible, is quite involved (see for instance [Matanga, 1993]); and reservoir displacements with dispersion, gravity and compositional effects can be modeled.

We show examples of incompressible, unstable miscible flow with gravity and dispersion, waterflooding, and compositional displacements, and compare them with conventional twodimensional simulations. The agreement is excellent and the streamline method is typically 100 10,000 times faster. We conclude by discussing the potential of this technique to predict reservoir production and its uncertainty from an ensemble of geostatistical descriptions of flow properties on of order $10^{6}$ to $10^{8}$ grid blocks.

\section{Theory}

Consider the equation for conservation of mass of some species $i=1, n$ :

$$
\frac{\partial M_{i}}{\partial t}+\underline{\nabla} \cdot \underline{F_{i}}=0
$$

where $M_{i}$ is the mass of $i$ per unit volume and $E_{i}$ is the mass flux per unit area, and $\frac{\partial}{\partial t} \sum_{i=1}^{n} M_{i}=0$, since mass is conserved. We assume that from Darcy's law we can relate $E_{i}$ to a pressure gradient and that given the distribution of composition, $M_{i}(x)$, at some time $t$, we can compute the pressure and fluxes $E_{i}$.

We further assume that we know a onedimensional solution to equation (1) (this may be done analytically, numerically or experimentally) $M_{i}^{1}(x, t)$ that obeys

$$
\frac{\partial M_{i}^{1}(x, t)}{\partial t}+\frac{\partial F_{i}^{1}}{\partial x}=0,
$$

where $F_{i}^{1}$ is the mass flux for the one-dimensional problem. Later we will discuss appropriate boundary and initial conditions for this solution.

The idea then, is to find a vector $\underline{v}$ such that equation (1) can be rewritten as:

$$
\frac{\partial M_{i}}{\partial t}+\underline{v} \cdot \underline{\nabla} F_{i}^{1}=0
$$

It is always possible to do this, although $\underline{y}$ may be a function of composition and composition gradient.

We then define a generalized time-of-flight $\tau$ by

$$
\frac{\partial}{\partial \tau} \equiv \underline{v} \cdot \underline{\nabla}
$$


If we define $\xi$ as a coordinate parallel to $\underline{v}$, then equation (4) is equivalent to $\partial \tau / \partial \xi=1 /|\underline{v}|$. Then equation (3) becomes

$$
\frac{\partial M_{i}}{\partial t}+\frac{\partial F_{i}^{1}}{\partial \tau}=0
$$

which is similar in form to equation (2). Thus the one-dimensional solution $M_{i}=M_{i}^{1}(\tau, t)$ traced along the generalized streamlines $\underline{v}$ will reproduce the full three-dimensional solution. $\underline{y}$ and $\tau$ can be computed from the known pressure field.

The method is thus, schematically, as follows:

(1) From an initial distribution of fluid $M_{i}(x, t)$ find the pressure field and component fluxes.

(2) Compute the time-of-flight along generalized streamlines for the entire computational domain.

(3) Assign a composition $M_{i}^{l}(\tau, t+\Delta t)$ to a grid block having time-of-flight $\tau$. This represents the new composition field at some time $t+\Delta$ $t$.

(4) Return to step 1 to recompute the pressure field at time $t+\Delta t$.

The main assumption is that the composition along a streamline follows a one-dimensional solution. This means that the one-dimensional solution must have the same boundary and initial conditions as the three-dimensional problem. The boundary conditions would be the pressures and flow rates at the wells and the initial condition would be representative of the whole reservoir. We further assume that these boundary conditions remain the same, even when we update the streamlines. In the examples we present, we have fixed flow rates and pressures at inlet and outlet and constant initial conditions. For these cases, the method works well. For more complex examples, it may be difficult, or impossible, to find a single one-dimensional solution that is representative of the flow everywhere in the reservoir. Moreover, we have implicitly assumed that the flow follows the instantaneous streamlines. For cases where gravity or cross-flow processes are dominant, this may be a poor approximation and the fluid composition along a streamline in three dimensions may be very different from the composition in a one-dimensional problem. A full discussion of these issues will be the subject of further research.

We illustrate the method with an example: twophase incompressible flow with gravity. Equation (1) for conservation of water is equivalent to

$$
\phi \frac{\partial S_{w}}{\partial t}+\underline{v}_{t} \cdot \underline{\nabla} f_{w}+\left(\rho_{w}-\rho_{o}\right) \underline{g} \cdot \underline{\nabla} G=0,
$$

where $\underline{\nu}_{t}$ is the total velocity, $f_{w}$ is the fractional flow of water and $G=\lambda_{o} \lambda_{w} /\left(\lambda_{o}+\lambda_{w}\right)$ where $\lambda_{o}$ and $\lambda_{w}$ are the oil and water mobilities respectively. This is a standard conservation equation [Lake, 1989]. Let us assume that we will consider waterflooding in a reservoir where the principal flow direction is horizontal. Thus we have a representative one-dimensional solution for $S_{w}^{1}(x, t)$ that obeys

$$
\phi \frac{\partial S_{w}^{1}}{\partial t}+v_{t} \frac{\partial f_{w}^{1}}{\partial x}=0,
$$

which is the Buckley-Leverett equation without gravity. Then we can re-write equation (6) as

$$
\phi \frac{\partial S_{w}}{\partial t}+\underline{v} \cdot \underline{\nabla} f_{w}
$$

by letting

$$
\underline{v}=\underline{v}_{t}+\left(\rho_{w}-\rho_{o}\right) \underline{g} \frac{\partial G\left(S_{w}\right)}{\partial f_{w}\left(S_{w}\right)} .
$$

Defining $v_{t} \partial / \partial \tau=\underline{v} \cdot \underline{\nabla}$ we find

$$
\phi \frac{\partial S_{w}}{\partial t}+v_{t} \frac{\partial f_{w}}{\partial \tau}=0
$$

and we propagate the Buckley-Leverett solution $S_{w}^{1}(\tau, t)$ along each streamline, defined by $\underline{y}$ :

If we ignored gravity in multiphase flow, $\underline{v}=\underline{v}_{t}$ and the technique reduces to the normal tracing of streamlines which is equivalent to the periodic updating of streamtubes previously presented [Thiele et al., 1995]. In all the cases we present here $\underline{v}=\underline{v}_{t}$, but the general theory has been developed to demonstrate the power and general applicability of the method.

The technique we use to trace streamlines and record the time-of-flight is described elsewhere [King et al., 1993]. When we follow the streamlines through the system, we record a timeof-flight for each grid block. To propagate a onedimensional solution along streamlines we do the following: if the time is $t$ and the time-of-flight for a grid block is $\tau$, we assign it a composition $M_{i}^{1}(\tau, t)$. We also record the total mobility associated with this composition. Then, when we want to recompute the streamlines, we use these total mobilities to compute the pressure field, using standard numerical techniques. From the pressure field we can find the velocities and from this we can follow streamlines and recalculate new values of the time-of-flight at each grid block. This is no more complicated for a compositional displacement than 
for tracer flow, once we have tabulated the onedimensional solution.

\section{Tracer Flow}

The streamline method is exact for tracer flow, except for the approximations inherent in the computation of the velocity field. The onedimensional solution for the tracer concentration, $c$, that is traced along a streamline is simply a step function: $c=1$ for $\tau \leq t$ and $c=0$ for $\tau t$. Figure 1 shows a simulation of tracer flow through a heterogeneous two-dimensional domain. There is a constant flow rate on the left-hand boundary and constant pressure at the outlet. The results are compared with conventional simulation using Eclipse, a commercial reservoir simulator that uses single point upstream weighting to solve the component conservation equations [1993a], and a research code developed at BP that uses a high order method - flux corrected transport - to solve the conservation equations [Christie, 1989; Christie and Bond, 1987]. Both these simulations suffer from numerical diffusion that tends to smear the profile over several grid blocks, although the BP code gives superior results to Eclipse. This demonstrates that the streamline method provides
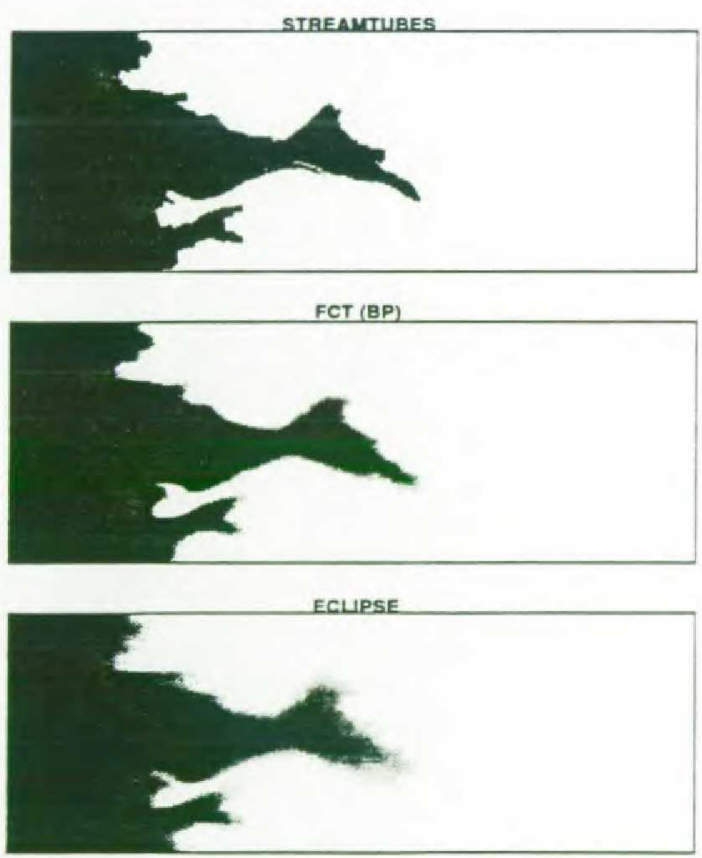

Figure 1. Tracer flow through a heterogeneous domain at 0.3 pore volumes injected. The simulation uses 250 by 100 grid blocks, the dimensionless correlation length of the permeability field is 0.3 and the variance in the logarithm of permeability is 1 The results using streamlines is compared with a BP code that uses flux corrected transport (FCT) to solve the component conservation equation, and Eclipse, a commercial reservoir simulator that uses single point upstream weighting. Flow through the same permeability field is shown in Figures 5 and 7. solutions that are free from numerical diffusion and thus, for advectively dominated problems, can resolve features down to the grid block scale.

\section{Tracer Flow with Dispersion}

Longitudinal dispersion can be included in the streamline method by tracing a one-dimensional solution to the advection/dispersion equation along each streamline [Thiele et al., 1994a]. Transverse dispersion, however, is more difficult to model, since it represents the transfer of fluid in a direction perpendicular to the total velocity. Previous streamline methods have been unable to account for any transverse flow processes. In this section we describe a method that incorporates both transverse and longitudinal dispersion by borrowing ideas from particle tracking. For more details on particle tracking the reader is referred to [Tchelepi and Orr, 1994].

As the streamline is traced through each grid block, dispersion is included by adding a random component to its position in a direction both longitudinal and transverse to the direction of flow. The random displacement has a Gaussian distribution and is proportional to the local flow velocity and the input dispersivity. These perturbations will alter the computed time-of-flight compared to a calculation without dispersion. A step function one-dimensional solution for the tracer concentration is mapped along each streamline as before. However, we trace each streamline several times with different random displacements. The concentration of a grid block is the average of the concentrations from the different sets of streamlines. It can be proved that this method will, if each streamline is traced a sufficient number of times, reproduce solutions to the advection/dispersion equation [Tchelepi and $\mathrm{Orr}$, 1994].

We define a Peclet number, $P_{e}$ as follows: $P_{e}=L / \alpha$, where $L$ is the length of the system and $\alpha$ is the (longitudinal) dispersivity. Figure 2 shows three comparisons of the streamline method with the BP program for tracer flow in a heterogeneous medium at different Peclet numbers. The transverse and longitudinal dispersivities are equal, although we can, in general, make them different. When the physical dispersion dominates over numerical diffusion, the results of the two codes are essentially identical. The simulations are performed on 100 by 200 grid blocks. In Figure 2, each streamline is traced once to generate the top picture, six times for the middle and ten times for the bottom picture. These figures demonstrate that the streamline method can successfully account for both longitudinal and transverse dispersion in single phase flow. 


\section{Tracer Flow with Gravity}

In this section we simulate the injection of a lighter fluid into a denser fluid of the same viscosity in a vertical two-dimensional system. There is a constant potential at both inlet and outlet boundaries and no flow at the top and bottom. Here the velocity field does change with time, since the injected fluid, being buoyant, will tend to rise to the top of the domain. As before we trace a step function concentration profile along streamlines. The streamlines are now periodically recomputed. We define a gravity number, $N_{g}=L k g \Delta \rho / H q \mu_{o}$, where $L$ is the system length, $H$ is the height, $\Delta \rho$ is the density difference between the fluids, $q$ is the Darcy velocity, $\mu_{o}$ is the oil viscosity and $k$ is the average permeability. Typical values of $N_{g}$ are in the range 0.001 to 10 for reservoir flows. Figure 3 illustrates flow at two gravity numbers (with no dispersion) compared with the BP program. With no gravity, shown in Figure 2, the main flow channel is along the bottom of the system. Notice that the streamline method successfully predicts the tendency for the injected fluid to flow along the top of the system as we increase the gravity number. The streamlines are recomputed up to eighty times during the displacement to reach breakthrough. This is approximately 20 to over 100 times faster than the BP code, depending on the gravity number. The streamline method can successfully account for gravity in miscible displacements for this case.

\section{Unstable Miscible Flow with Gravity and Dispersion}

The next examples are for first contact miscible flow, where a fluid of lower viscosity is injected. In this case the velocity field changes with time due to both buoyancy and viscous effects. As before, streamlines following the total velocity are traced through the system.

The flow suffers from a viscous instability, and to obtain stable solutions for the streamline method it is necessary mitigate this instability at the small scale. In our previous work this was done by incorporating a viscous fingering model [Thiele et al., 1994a; Thiele et al., 1995]. Here, in order to obtain accurate comparisons with conventional
Streamline
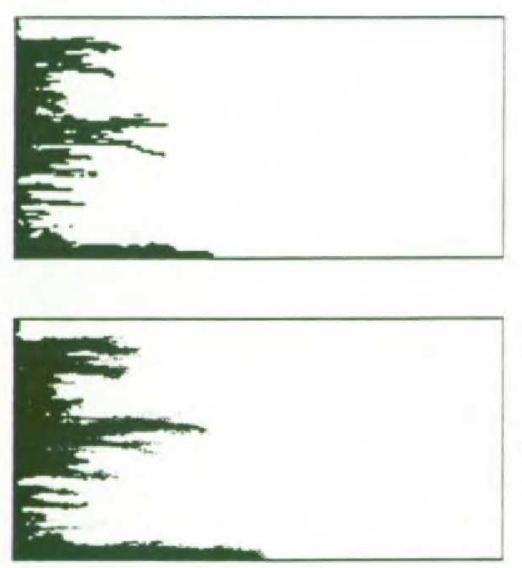

$P_{e}=20000.0$

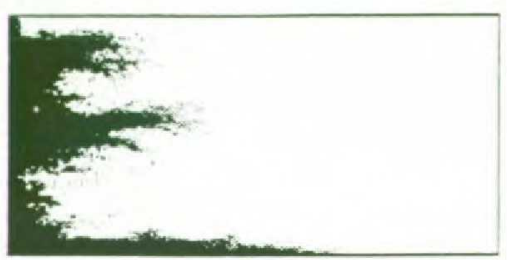

BP code
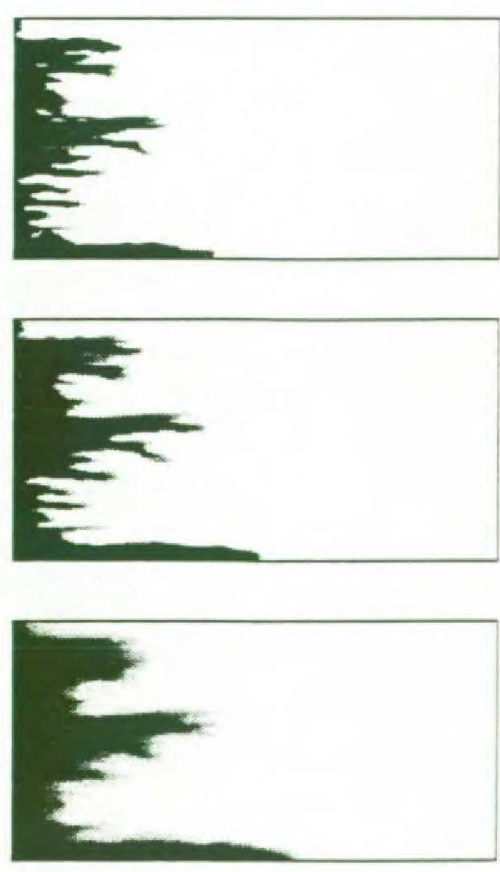

Figure 2. The streamline method compared with conventional finite difference simulation for tracer flow at different Peclet numbers. The permeability field has dimensionless correlation lengths of 1 and 0.1 in the longitudinal and transverse directions respectively. The logarithm of the permeability has a variance of 1.87 . Flow through the same permeability field is shown in Figures 3 and 4. 
simulation, we add a small amount of dispersion, which approximately represents the numerical diffusion in the BP code. We perform the streamline simulations with a longitudinal Peclet number of 20,000 and a transverse Peclet number of 200,000 . As for the tracer case, the onedimensional solution that is mapped along each streamline is simply a step-function in concentration.
Figure 4 shows comparisons of the streamline method with high resolution simulation using the BP code for miscible flow with a mobility ratio of 10. Again the agreement is excellent. However, the streamline method required only 3 updates of the pressure field to recompute the streamlines, as against several thousand updates for the conventional method. This equates to a 1,000 fold saving in computer time.
Streamline
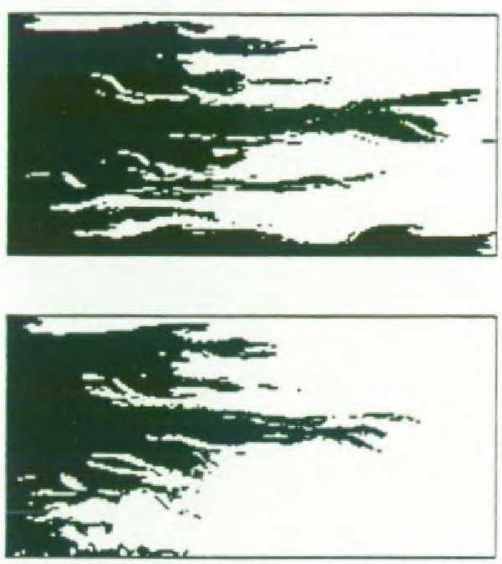

BP code
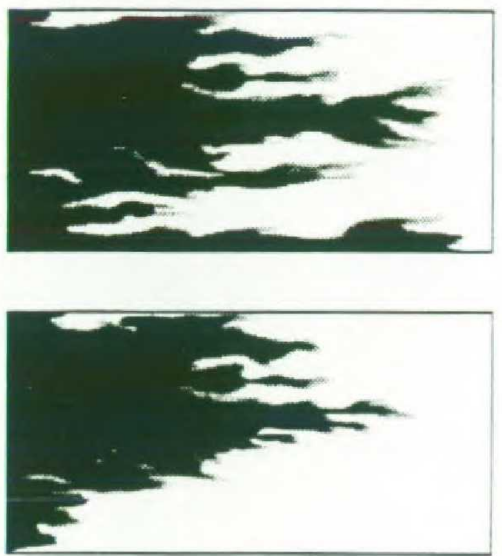

Figure 3. Tracer flow using streamlines compared with conventional simulation for two different gravity numbers. The upper picture is at 0.5 pore volumes injected and the lower is at 0.35 pore volumes injected.

\section{Streamline}
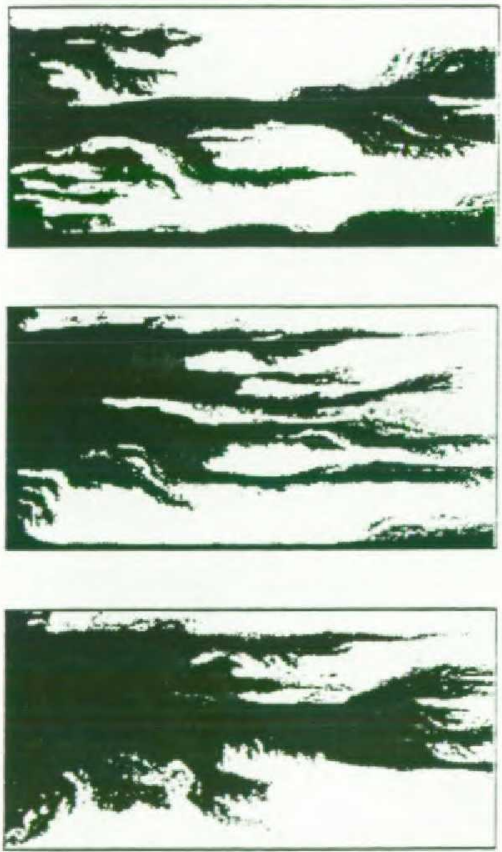

$$
\mathrm{N}_{\mathrm{g}}=0.0
$$$$
N_{g}=2.0
$$

$N_{g}=10.0$
BP code
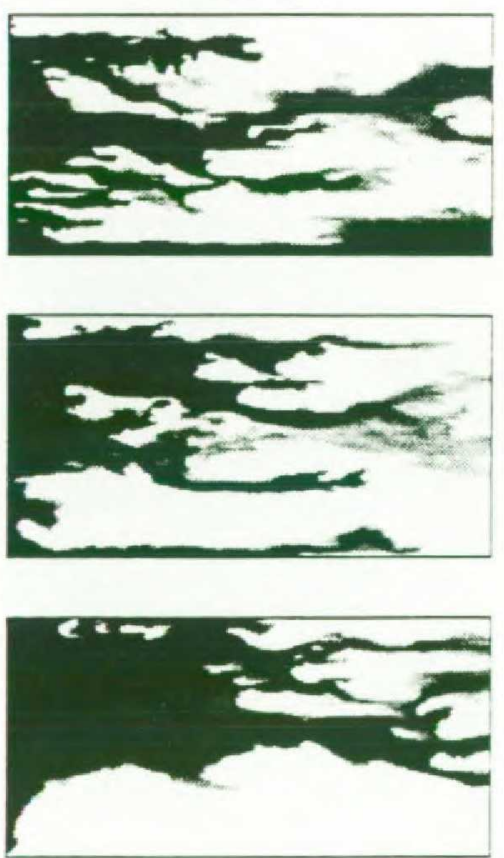

Figure 4. The streamline method compared against conventional simulation for unstable miscible displacements with gravity and dispersion at 0.6 pore volumes injected. 


\section{Two Phase Flow}

We will now present results for multiphase flow [Thiele et al., 1995]. We will ignore the effects of gravity and dispersion. As before we trace streamlines that follow the total velocity. The difference is now is that we propagate a onedimensional solution to a multiphase conservation equation along each streamline. Once we know the one-dimensional solution, this is, in principle, no more difficult than the single phase examples described previously.

In this section we present predictions for waterflooding. The solution to the BuckleyLeverett equation, (7), with constant initial and boundary conditions, is propagated along each streamline. Figure 5 shows a comparison of the streamline method with conventional simulation using Eclipse. The results are similar, except that the streamline code does not suffer from numerical diffusion and ran almost 100 times faster. Figure 6 compares recovery curves for waterflooding at different oil/water viscosity ratios compared against simulations using Eclipse. The agreement is very good in all cases.
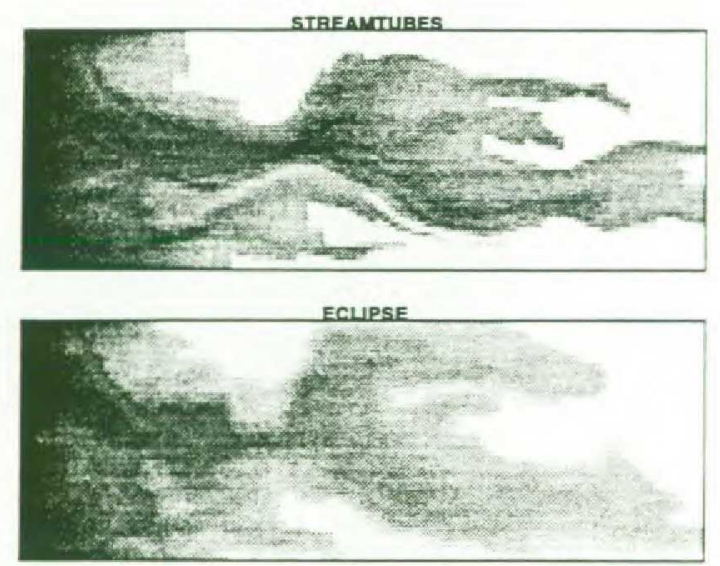

Figure 5. The streamline method compared with conventional simulation using Eclipse for waterflooding at 0.3 pore volumes injected. The water saturation is shown.

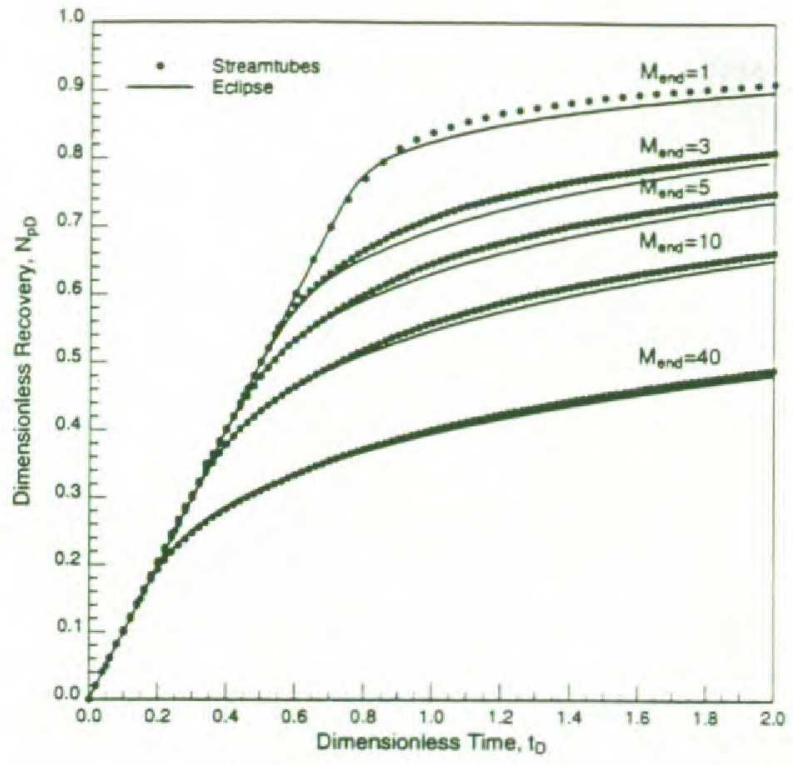

Figure 6. Waterflood oil recovery curves for displacements in a heterogeneous cross-section at different end point mobility ratios $M_{\text {end }}$. The predictions using the streamline method are compared with those using Eclipse.

\section{Compositional Displacements}

The efficiency of the streamline method becomes apparent when we consider compositional displacements [Thiele et al., 1995]. The first step is to find a representative one-dimensional solution that we will propagate along streamlines.

We use UTCOMP, a simulator developed at the University of Texas [1993b], to model a four component condensing/vaporizing gas drive. First we run a 500 block simulation in one dimension. We record the component concentrations, the gas saturation and the total mobility as a function of distance and time [Thiele et al., 1995].

The one-dimensional solution is then traced along streamlines. For every 0.2 pore volumes of gas injected, we recompute the streamlines. We know the total mobility in the system, which enables us to solve for the pressure field. From this we find the velocities and hence we can trace streamlines for the new flow field.

We compare the predictions of the streamline method with a two-dimensional finite difference computation using UTCOMP. The results are shown in Figure 7. The streamline method correctly identifies the main flow directions and honors the sequence of fluid fronts. The UTCOMP solution suffers from a significant amount of numerical diffusion which couples with the phase behavior to reduce the apparent mobility contrast in the flood [Thiele et al., 1995]. 
The major approximation of the streamline method in this case is to assume that all the relevant phase behavior is captured in the representative onedimensional solution. We have decoupled the full, multidimensional flow and flash calculations into a series of similar one-dimensional problems. This leads to a significant saving in computer time - the streamline method was almost 10,000 times faster than UTCOMP in this example.

\section{Three Dimensional Flow}

Figure 8 shows an example three-dimensional problem solved using the streamline method. Here a miscible fluid is injected across one face of a heterogeneous rectangular domain containing over 300,000 grid blocks. This problem was too large to run a comparison with conventional simulation using our current computer resources, but illustrates how the method described here can be readily extended to three dimensions.
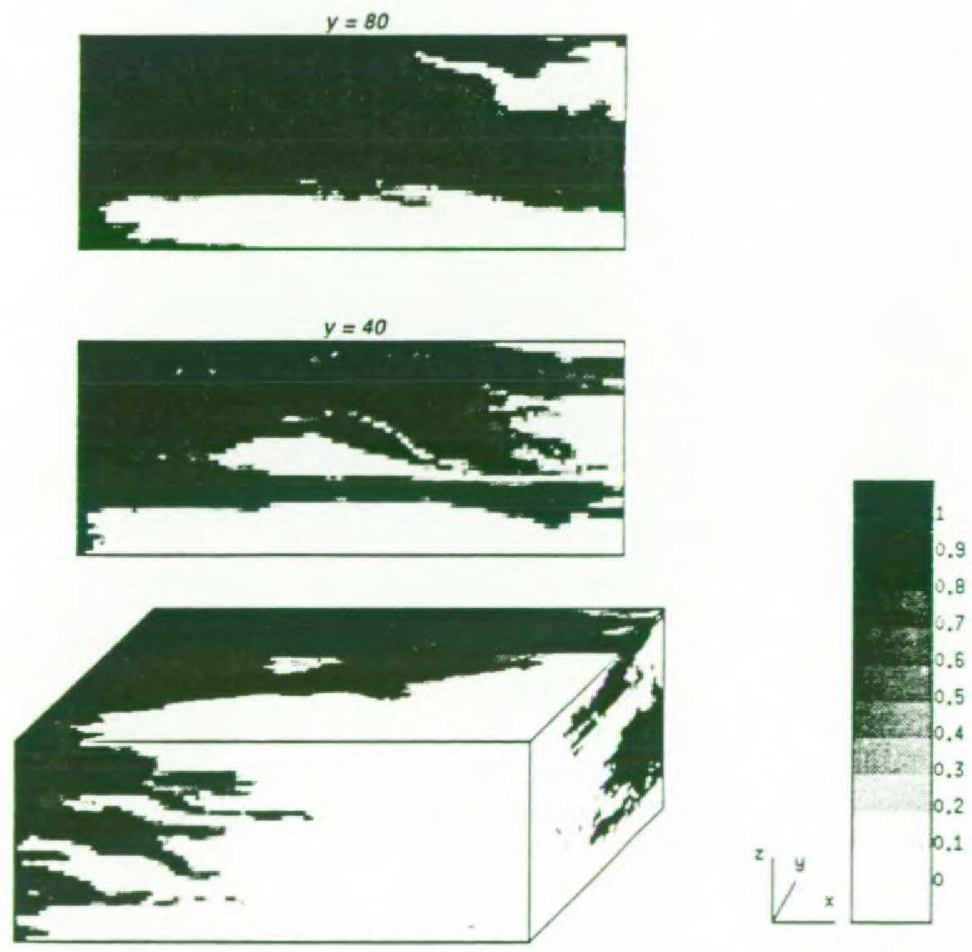

Figure 8. Three-dimensional streamlines used to simulate a miscible displacement with a mobitity ratio of 1.1 through a heterogeneous field containing 320,000 grid blocks.
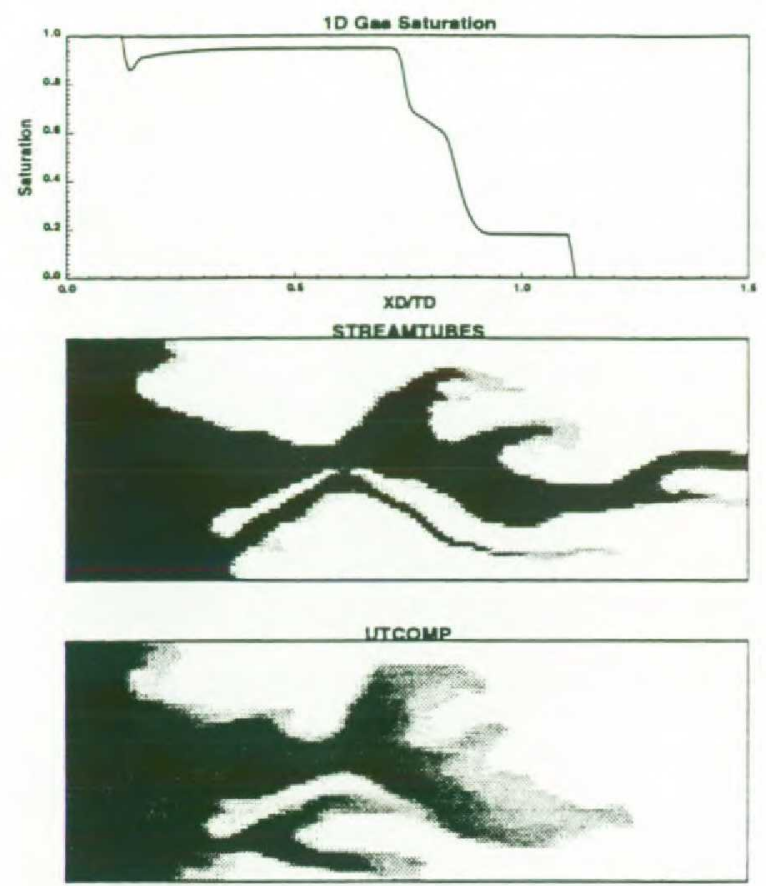

Figure 7. The streamline method compared with compositional simulation for a condensing/vaporizing gas drive. The top figure shows the one-dimensional gas saturation that is mapped along each streamline to produce the two-dimensional streamline profile shown.
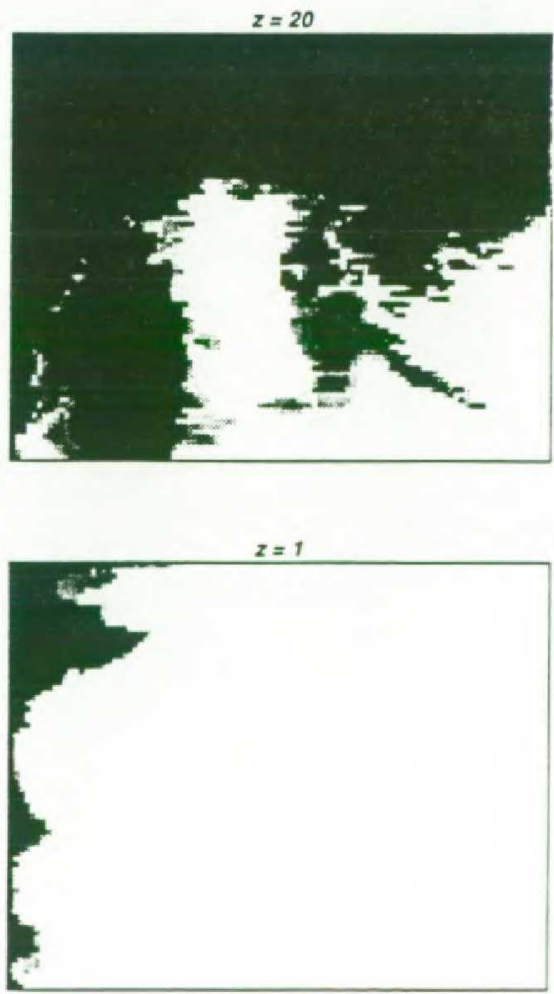


\section{Discussion}

Once we have a one-dimensional solution, the streamline method is only limited by the size of problem for which the pressure equation can be solved. We are currently working on a code that can simulate reservoir flows with multiple wells with 1 to 2 million grid blocks on a workstation. The use of parallel computers should enable problems at least ten times larger to be solved. This offers the promise for rapid estimation of reservoir recovery based on fine scale geostatistical data without the need to upscale, while honoring, albeit approximately, the physics of flow.

\section{Conclusions}

We have developed a theory of generalized streamlines that can decouple any three-dimensional reservoir flow into a series of one-dimensional problems. We illustrated the method by comparing two-dimensional predictions against conventional simulation. For all the cases studied - tracer flow with and without gravity and dispersion, unstable miscible flow, waterflooding and compositional displacements - the streamline method produced accurate results, free from numerical diffusion, up to 10,000 times faster than conventional methods.

We discussed how the method could be used to model multi-million grid block problems that go from a detailed geostatistical realization to reservoir performance prediction without the need for upscaling.

\section{Acknowledgments}

We thank Thomas Hewett for many useful discussions, British Petroleum for allowing us to use their codes, the Department of Petroleum Engineering at the University of Texas for the use of UTCOMP, and the Stanford University Gas Injection Industrial Affiliates Program, the Stanford Center for Reservoir Forecasting and British Petroleum for financial support.

\section{References}

Eclipse 100 Reference Manual. Intera Information Technologies Limited, Henley-on-Thames, UK, 1993a. 93a edition.

UTCOMP-3.2: An Equation of State Compositional Simulator. Center for Petroleum and Geosystems Engineering, University of Texas at Austin, TX $78712,1993 b$.

Bear, J., Dynamics of Fluids in Porous Media, Dover Publications Inc, New York, 1972.

Blunt, M. J., J. W. Barker, B. Rubin, M. Mansfield, I. D. Culverwell, and M. A. Christie,
Predictive Theory for Viscous Fingering in Compositional Displacement, SPE Reservoir Engineering, (February), 73-80, 1994.

Christie, M. A., High Resolution Simulation of Unstable Flows in Porous Media, SPE Reservoir Engineering, 4, 297-304, 1989.

Christie, M. A., and D. J. Bond, Detailed Simulation of Unstable Processes in Miscible Flooding, SPE Reservoir Engineering, 2, 514522, 1987.

Christie, M. A., A. H. Muggeridge, and J. J. Barley, A Model Study of Viscous Fingering, SPE Reservoir Engineering, (February), 19-26, 1993.

Emanuel, A. S., G. K. Alameda, R. A. Behrens, and T. A. Hewett, Reservoir Performance Prediction Methods on Fractal Geostatistics, SPE Reservoir Engineering, (August), 311-318, 1989.

Hewett, T., and R. Behrens, Scaling Laws in Reservoir Simulation and Their Use in a Hybrid Finite Difference/Streamtube Approach to Simulating the Effects of Permeability Heterogeneity, In Reservoir Characterization II, edited by L Lake and J Carroll, Academic Press Inc, London, 1991.

Higgins, R. V., and A. J. Leighton, A Computer Method to Calculate Two-Phase Flow in Any Irregularly Bounded Porous Medium, Journal of Petroleum Technology, (June), 679-683, 1962a.

Higgins, R. V., and A. J. Leighton, Computer Prediction of Water Drive of Oil and Gas Mixtures Through Irregularly Bounded Porous Media Three Phase Flow, Journal of Petroleum Technology, (September), 1048-1054, 1962b.

King, M. J., M. J. Blunt, M. Mansfield, and M. A. Christie, Rapid Evaluation of the Impact of Heterogeneity on Miscible Gas Injection, Proceedings of the Western Regional Meeting, SPE 26079, Anchorage, Alaska, 1993.

Lake, L. W., Enhanced Oil Recovery, PrenticeHall, Englewood Cliffs, 1989.

Lake, L. W., J. R. Johnston, and G. L. Stegemeier, Simulation and Performance Prediction of a Large-Scale Surfactant/Polymer Project, Society of Petroleum Engineers Journal, (December), 731-739, 1981.

Matanga, G. B., Stream Functions in ThreeDimensional Groundwater Flow, Water Resources Research, 29(9), 3125-3133, 1993.

Renard, G., A 2D Reservoir.Streamtube EOR Model with Periodical Automatic Regeneration of Streamlines, In Situ, 14(2), 175-200, 1990. 
Tchelepi, H., and F. M. Orr, Interaction of Viscous Fingering, Permeability Heterogeneity and Gravity Segregation in Three Dimensions, SPE Reservoir Engineering, 9, 266-271, (November)1994.

Thiele, M. R., M. J. Blunt, and F. M. Orr, A New Technique for Predicitng Flow in Heterogeneous Systems Using Streamtubes, Proceedings of the SPE/DOE Ninth Symosium on Improved Oil Recovery, SPE 27834, Tulsa, OK, 1994a.

Thiele, M. R., M. J. Blunt, and F. M. Orr, Predicting Multicomponent, Multiphase Flow in Heterogeneous Systems using Streamtubes, Proceedings of the 4th European Conference on the Mathematics of Oil Recovery, Roros, Norway, 1994b.

Thiele, M. R., M. J. Blunt, and F. M. Orr, Modeling Flow in Heterogeneous Media Using Streamtubes - Parts I and 2, In Situ (submitted), 1995. 